\title{
State Estimation Based on Multirate Kalman Filter for Power Systems Driven by Switching Inverter
}

\author{
Hiroki Kurumatani* ${ }^{*}$ Member, Seiichiro Katsura* Senior Member
}

(Manuscript received May 2, 2018, revised Oct. 29, 2018)

\begin{abstract}
This paper presents a design methodology of a Kalman filter for a multirate control system with a fast input system. There are many applications whose sensory system is slower than the input system in a power system. The sensory system is required to perform fast sensing while ensuring a low signal-to-noise ratio, although it requires additional time compared with that of a low-resolution converter. The Kalman filter is an effective tool in such a situation and it estimates parameters while eliminating noise. Because the optimality of this filter is ensured when the system model and noise variance on the system are well identified, rigorous discretization should be considered on the multirate control system. With this in mind, the paper presents the design method of a multirate Kalman filter as is the case for a single-rate controller. Further, the noise variance and oversampling ratio of the multirate controller is confirmed using the Monte Carlo method. Numerical simulation of a voltage inverter theoretically validates the design methodology.
\end{abstract}

Keywords: multirate system, Kalman filter, power electronics

\section{Introduction}

Power systems are widely used for industrial field. A motor drive system and a power management system with high performance are now necessities of our lives. As a high performance and efficiency in a power management are demanded, a feedback controller becomes an essential tool and importance of sensor systems significantly increases. Furthermore, wide spread and rapid development of an internet technology has led to change of industrial and system management structures and hence sensing process will plays a lot of significant roles. Such sensing-then-sharing process is a part of a framework such as the industry 4.0 and further demands for the sensor system will appear along with this rapidly-evolving framework.

For the sensor system, there is a problem that it has tradeoff between conversion speed and accuracy, namely resolutions in a time-domain and a spatial-domain. For example, a successive approximation (SAR) A/D converter performs a high-speed operation but provide moderate resolution, while a delta-sigma type and double-integral A/D converter provides high precision at low-speed operation ${ }^{(1)}$. When precision is required in sensing, its sampling rate is decreased. However, an input system of the power systems such as a switching inverter is tend to increase its switching frequency to achieve a high performance and efficiency while boosted by evolving power devices ${ }^{(3)(4)}$. Consequently, sampling times of the input system and the sensor system are different and hence multirate controller-designed is required. Certainly, the feedforward control for comparatively low-frequency switching inverter with using an exact-linearization is a powerful technique for the industrial

\footnotetext{
${ }^{*}$ Department of System Design Engineering, Keio University 3-14-1, Hiyoshi, Kohoku-ku, Yokohama 223-8522, Japan
}

field but the switching frequency should be high when the disturbance-suppression is required ${ }^{(2)}$. Such design is able to be simplified by putting an assumption that the input system works ideally. This technique is often used in motor drive applications while assuming ideal current and torque control. For this trend, a research ${ }^{(5)}$ designed systems with rigorous discretization and a research ${ }^{(6)}$ shows the relation between a bandwidth of a current controller and a practical limitation on a total performance. Basic-performance improvement of the input system is also considered ${ }^{(7)}$.

In contrast to improving the input system, fundamental solutions from the view point of the sensor system have been widely researched as well. A Kalman filter is a good tool for state estimation when system noise appears on an output of the sensor system. This filter is one of the optimal filters which minimize a variance of observation error. That is, a Kalman filter is a less phase-lag filter with a certain noisesuppression performance. Note that this optimality is ensured when a system model and noise variance on the system is well identified and hence a rigorous system model should be derived. However, variance of a process noise is difficult to identify as it depends on the switching frequency of the input system. Further, because update of the Kalman filter uses the output of the sensor system, the variance of the process noise should be designed on the system, which is discretized with a sampling time of the sensor system. Then, the paper designs a multirate Kalman filter for the system with the fast input system and shows a design guideline of the noise variance as is the case for a single-rate controller. Note that there are many researches for the multirate Kalman filter to establish the sensor-fusion ${ }^{(8)-(10)}$, namely for multirate output systems, but few researches focusing on the design of the multirate input-output system. Now, this paper mainly focuses on a time-invariant power system which has the switching inverter as the input system. 
Approaches and contributions of the paper are as follows. The paper discretizes a system with sampling times of the input system and the sensor system and derives two discrete models. Then, the paper integrates the two models while using lifting method and represents the system like a single-rate controller. Such block representation of the multirate system has been reported in ${ }^{(11)}$. On this single-rate model, an equivalent noise variance is defined. Then, noise distributions of the switching inverter is investigated with a mind that noise histograms do not depends on the sampling frequency but width of quantizers. A validity of the theory, mainly about the equivalent noise variance, is verified numerically with using the Monte Carlo method. Numerical simulation of a voltage inverter verifies a theoretical validity and an applicability of the designed multirate Kalman filter for the power system.

\section{Multirate Control System}

In this paper, a state-space representation is used for filter design. The paper uses following sets of numbers; natural numbers $\mathbb{N}$, nonnegative integers $\mathbb{Z}$ and real numbers $\mathbb{R}$.

2.1 System Description This paper considers a $N$-th order $(N \in \mathbb{N})$ single-input-single-output continuous timeinvariant linear-stochastic system expressed as

$$
\begin{aligned}
\dot{x}(t) & =A_{\mathrm{ct}} x(t)+B_{\mathrm{ct}} u(t)+H_{\mathrm{ct}} v(t) \ldots \ldots \ldots \ldots \ldots \ldots \ldots \ldots \ldots \ldots \ldots \ldots \ldots \ldots \ldots \ldots \ldots \ldots \ldots \\
y(t) & =C_{\mathrm{ct}} x(t)+w(t), \cdots \ldots \ldots \ldots \ldots \ldots \ldots \ldots
\end{aligned}
$$

where $t \in \mathbb{R}, x \in \mathbb{R}^{N \times N}, u \in \mathbb{R}, y \in \mathbb{R}, v \in \mathbb{R}$ and $w \in \mathbb{R}$ are time, a state vector, an input, an output, process noise and observation noise. $A \in \mathbb{R}^{N \times N}, B \in \mathbb{R}^{N \times 1}, C \in \mathbb{R}^{1 \times N}$ and $H \in \mathbb{R}^{N \times 1}$ are a state matrix, an input matrix, an output matrix and a noise-shaping matrix. Superscript $\bigcirc_{c t}$ stands for parameters of the continuous-time domain. The paper puts an assumption that $v$ and $w$ meat following conditions.

$$
\begin{aligned}
& \mathrm{E}\{v(t)\}=\mathrm{E}\{w(t)\}=0 \\
& \mathrm{E}\left\{\left[\begin{array}{l}
v(t) \\
w(t)
\end{array}\right]\left[\begin{array}{ll}
v^{\mathrm{T}}(t+\varepsilon) & w^{\mathrm{T}}(t+\varepsilon)
\end{array}\right]\right\}=\left[\begin{array}{ll}
Q & 0 \\
0 & R
\end{array}\right] \delta(\varepsilon) .
\end{aligned}
$$

Here, E[· ] provides an expected value, $\delta$ stands for Dirac delta function and $\varepsilon \in \mathbb{Z}$. Now, the paper supposes a situation that samples per second of a sensor or an A/D converter are limited by conversion time. Putting sampling period of the sensory system $:=T_{\mathrm{r} 1}$, a state-space equation in discrete-time domain is expressed as

$$
\begin{aligned}
& \dot{x}[k+1]=A_{\operatorname{Tr} 1} x[k]+B_{\operatorname{Tr} 1} u[k]+H_{\operatorname{Tr} 1} v[k] \cdots \cdots \\
& y[k]=C_{\operatorname{Tr} 1} x[k]+w[k] \text {, } \\
& A_{\mathrm{Tr} 1}=e^{A_{\mathrm{ct}} T_{\mathrm{r} 1}}, B_{\mathrm{Tr} 1}=\int_{0}^{\mathrm{T}_{\mathrm{r} 1}} e^{A_{\mathrm{ct}} \tau} d \tau B_{\mathrm{ct}} \\
& C_{\operatorname{Tr} 1}=C_{\mathrm{ct}}, H_{\operatorname{Tr} 1}=\int_{0}^{\mathrm{T}_{\mathrm{r} 1}} e^{A_{\mathrm{ct}} \tau} d \tau H_{\mathrm{ct}} .
\end{aligned}
$$

In above equations, the paper puts $x\left(k T_{\mathrm{r} 1}\right):=x[k](k \in \mathbb{Z})$. Here, the process noise is difficult to define when an update rate of the input signal is different from that of the sensory system. In power systems, an input system using a switching inverter is much faster than the sensory systems. Using a control period of the inverter system $T_{\mathrm{r} 2}$, the system is rediscretized as

$$
\begin{aligned}
& \dot{x}[k, l+1]=A_{\operatorname{Tr} 2} x[k, l]+B_{\operatorname{Tr} 2} u[k, l]+H_{\operatorname{Tr} 2} v[k, l] \cdots \\
& y[k]=C_{\operatorname{Tr} 2} x[k]+w[k] \text {, } \\
& A_{\mathrm{Tr} 2}=e^{A_{\mathrm{ct}} T_{\mathrm{r} 2}}, B_{\mathrm{Tr} 2}=\int_{0}^{\mathrm{T}_{\mathrm{r} 2}} e^{A_{\mathrm{ct}} \tau} d \tau B_{\mathrm{ct}} \\
& C_{\mathrm{Tr} 2}=C_{\mathrm{ct}}, H_{\mathrm{Tr} 2}=\int_{0}^{\mathrm{T}_{\mathrm{r} 2}} e^{A_{\mathrm{ct}} \tau} d \tau H_{\mathrm{ct}} .
\end{aligned}
$$

Here, the paper puts $x\left(k T_{\mathrm{r} 1}+l T_{\mathrm{r} 2}\right):=x[k, l](l \in \mathbb{Z})$. By setting $T_{\mathrm{r} 1}=n T_{\mathrm{r} 2}(n \in \mathbb{N}), x[k, n]$ is equal to $x[k+1]$. Then, state transition with the time step $T_{\mathrm{r} 1}$ is expressed as

$$
\begin{aligned}
& x[k+1]=A_{\operatorname{Tr} 1} x[k]+B_{\mathrm{m}} u_{\mathrm{m}}[k]+H_{\mathrm{m}} v_{\mathrm{m}}[k] \cdots \cdots \\
& y[k]=C_{\operatorname{Tr} 1} x[k]+w[k], \cdots \cdots \cdots \cdots \\
& B_{\mathrm{m}}=\left[\begin{array}{llll}
B_{\operatorname{Tr} 2} & A_{\operatorname{Tr} 2} B_{\operatorname{Tr} 2} & \cdots & A_{\operatorname{Tr} 2}^{n-1} B_{\operatorname{Tr} 2}
\end{array}\right] \cdots \cdots \cdots \\
& H_{\mathrm{m}}=\left[\begin{array}{llll}
H_{\operatorname{Tr} 2} & A_{\operatorname{Tr} 2} H_{\operatorname{Tr} 2} & \cdots & A_{\operatorname{Tr} 2}^{n-1} H_{\operatorname{Tr} 2}
\end{array}\right] \cdots \cdots \cdots \\
& u_{\mathrm{m}}[k]=\left[\begin{array}{lll}
u[k, n-1] & \cdots & u[k, 0
\end{array}\right]^{\mathrm{T}} \cdots \cdots \cdots \cdots \\
& v_{\mathrm{m}}[k]=\left[\begin{array}{lll}
v[k, n-1] & \cdots & v[k, 0
\end{array}\right]^{\mathrm{T}} \ldots \ldots \cdots \cdots
\end{aligned}
$$

Here, subscript $\bigcirc_{\mathrm{m}}$ stands for parameters of the multirate model, $B_{\mathrm{m}} \in \mathbb{R}^{N \times n}, H_{\mathrm{m}} \in \mathbb{R}^{N \times n}, u_{\mathrm{m}} \in \mathbb{R}^{n \times 1}$ and $v_{\mathrm{m}} \in \mathbb{R}^{n \times 1}$. This form is able to expresses the noises of both the input system and the sensor system with their control period. Simultaneously, above representation takes a form of a single-rate multi-input-single-output system. Considering the assumption expressed as (4), a redefined process noise $H_{\mathrm{m}} v_{\mathrm{m}}[k]=$ : $v_{\mathrm{hm}}[k]$ meets a following condition.

$$
\begin{array}{r}
\mathrm{E}\left\{v_{\mathrm{hm}}[k]\right\}=0 \ldots \ldots \ldots \ldots \ldots \ldots \ldots \\
\mathrm{E}\left\{[ \begin{array} { c } 
{ v _ { \mathrm { hm } } [ k ] } \\
{ w [ k ] }
\end{array} ] \left[\begin{array}{ll}
v_{\mathrm{hm}}^{\mathrm{T}}[k+\varepsilon] & \left.\left.w^{\mathrm{T}}[k+\varepsilon]\right]\right\} \\
& =\left[\begin{array}{cc}
H_{\mathrm{m}} Q H_{\mathrm{m}}^{\mathrm{T}} & 0 \\
0 & R
\end{array}\right] \delta(\varepsilon) . \cdots
\end{array}\right.\right.
\end{array}
$$

$H_{\mathrm{m}}$ is explicitly a matrix with damping functions since it contains the state matrix, whose norm decreases by repeated multiplication. Namely, an oversampling technique damps variances compared with a low sampling-rate controller. This effect was discussed numerically in a section 4 .

\section{Multirate Kalman Filter}

A Kalman filter is used to estimate a state vector while reducing an observation noise. Firstly, the paper introduces a classical single-rate Kalman filter. Considering the system represented as (5) and (6), the Kalman filtering process is composed of prediction of a state vector and an estimated error covariance matrix, modification of predicted state vector and estimated error covariance matrix, and update of a Kalman gain as

$$
\begin{aligned}
& \hat{x}_{\mathrm{p}}[k+1]=A_{\operatorname{Tr} 1} \hat{x}[k]+B_{\operatorname{Tr} 1} u[k] \cdots \cdots \cdots \cdots \\
& P_{\mathrm{p}}[k+1]=A_{\operatorname{Tr} 1} P[k] A_{\operatorname{Tr} 1}^{\mathrm{T}}+H_{\operatorname{Tr} 1} Q H_{\operatorname{Tr} 1}^{\mathrm{T}} \cdots \cdots \cdots \\
& \hat{x}[k+1]=\left(I_{n}-K C_{\operatorname{Tr} 1}\right) \hat{x}_{\mathrm{p}}[k+1]+K y[k] \cdots \cdots \\
& P[k+1]=\left(I_{n}-K C_{\operatorname{Tr} 1}\right) P[k+1] \cdots \cdots \cdots \cdots \\
& K=P_{\mathrm{p}}[k] C_{\operatorname{Tr} 1}\left(R+C_{\operatorname{Tr} 1} P_{\mathrm{p}}[k] C_{\operatorname{Tr} 1}^{\mathrm{T}}\right)^{-1}, \cdots \cdots \cdots \cdots
\end{aligned}
$$

where $\hat{x}_{\mathrm{p}}, \hat{x}, P, P_{\mathrm{p}}, K$, and $I_{n}$ are a predicted and modified state vector, predicted and modified estimated error covariance matrix, a Kalman gain, and an $n \times n$ identity matrix. 
Following filtering process is then derived as

$$
\hat{x}[k+1]=\left(I_{n}-G\right) \hat{x}_{\mathrm{p}}[k+1]+G\left(x[k]+C_{\operatorname{Tr} 1}^{-1} w[k]\right), \cdots
$$

where

$$
\begin{aligned}
G= & \left(H_{\operatorname{Tr} 1} Q H_{\operatorname{Tr} 1}^{\mathrm{T}}+A_{\operatorname{Tr} 1} P[k] A_{\operatorname{Tr} 1}^{\mathrm{T}}\right) \\
& \left(C_{\operatorname{Tr} 1}^{-T} R C_{\operatorname{Tr} 1}^{-1}+H_{\operatorname{Tr} 1} Q H_{\operatorname{Tr} 1}^{\mathrm{T}}+A_{\operatorname{Tr} 1} P[k] A_{\operatorname{Tr} 1}^{\mathrm{T}}\right)^{-1} .
\end{aligned}
$$

This equation denotes that the Kalman filter works as a lowpass filter whose cutoff frequency is determined by reliabilities of input and observation precision. Here, it should be noted that these reliabilities are defined by influence of the process noise and the observation noise at sampling point. Considering the block representation (13) and (14), the fast input changes the transition process of the input effort and noise. Then, the multirate Kalman filter is designed by only revising the prediction step as

$$
\begin{aligned}
& \hat{x}_{\mathrm{p}}[k+1]=A_{\operatorname{Tr} 1} \hat{x}[k]+B_{\mathrm{m}} u_{\mathrm{m}}[k] \cdots \cdots \\
& P_{\mathrm{p}}[k+1]=A_{\operatorname{Tr} 1} P[k] A_{\operatorname{Tr} 1}^{\mathrm{T}}+H_{\mathrm{m}} Q H_{\mathrm{m}}^{\mathrm{T}} .
\end{aligned}
$$

The lifted terms $B_{\mathrm{m}}$ and $H_{\mathrm{m}}$ dictate an influence of the input and noise to a state of a next step in the single-rate controller. In this process, ideal state transition caused by the input is rigorously identified by (28), while an effect of the process noise to the state at sampling point is tracked as shown in (29). Although the multirate Kalman filter has an oversampling effect in the input stage, the multirate Kalman filter is able to be designed in the same way as the single-rate one when the effects of these terms are obtained.

\section{Examination of Multirate Effect}

This section shows a multirate effect from theoretical and numerical view points. The advantage of the multirate controller is concentrated in (20). This equation shows that the multirate control has a capability to suppress the process noise. For this advantage, the prediction step in the filtering should be modified. Thus, the paper should verifies the influence of the multirate controller.

4.1 Reduction of Noise by Oversampling It is well known that the output distortion decreases as increasing the switching frequency of an inverter. This is because the fast switching scatters a noise power to a wide bandwidth. The Plancherel theorem denotes that the Fourier transform is unitary and then following equation is established;

$$
\sum_{\mathrm{k}=0}^{\mathrm{N}-1}|f[k]|^{2}=\frac{1}{\mathrm{~N}} \sum_{\mathrm{k}=0}^{\mathrm{N}-1}|F[k]|^{2},
$$

where $\mathrm{N}, f$ and $F$ are a number of samples, an optional continuous-signal and its discrete Fourier transformation. Here, $F$ can be approximately expressed by using the Fourier coefficients as

$$
F[k]=\sum_{\mathrm{p}=0}^{N-1} f[p] e^{-j 2 \pi k\left(\mathrm{p} T_{\mathrm{p}} / \mathrm{N}\right)} \approx \frac{N}{2} c_{\mathrm{k}}, \cdots \cdots \cdots \cdots
$$

where $c_{\mathrm{k}}$ is Fourier coefficient defined as

$$
c_{\mathrm{k}}=\frac{2}{\mathrm{~N} T_{\mathrm{s}}} \int_{0}^{\mathrm{N} T_{\mathrm{s}}} f(t) e^{-j 2 \pi \mathrm{k} t} d t,
$$

and $j$ is imaginary number, and $T_{\mathrm{s}}$ is sampling time. The Plancherel theorem then described as

$$
\frac{\mathrm{N}}{2} \sum_{\mathrm{k}=0}^{\mathrm{N} / 2-1}\left|c_{\mathrm{k}}\right|^{2} \approx \sum_{\mathrm{k}=0}^{\mathrm{N}-1}|F[k]|^{2} .
$$

Here, when a noise distribution is invariable regardless of the sampling frequency and the signal is strictly stationary, following equation is established;

$$
\sum_{\mathrm{k}=0}^{\mathrm{nN}-1}|F[k]|^{2} \approx n \sum_{\mathrm{k}=0}^{\mathrm{N}-1}|F[k]|^{2}, \cdots \cdots \cdots \cdots \cdots \cdots \cdots
$$

where $\mathrm{n} \in \mathbb{N}$ is an optional number. When increasing the sampling frequency $\mathrm{n}$ times, a number of samples also increase $\mathrm{n}$ times. Assuming a case where measuring a signal in $\mathrm{N} T_{\mathrm{s}}$ and sampling data at intervals of $T_{\mathrm{s}}$ and $T_{\mathrm{s}} / \mathrm{n}$, following equations are established;

$$
\begin{aligned}
& \frac{\mathrm{N}}{2} \sum_{\mathrm{k}=0}^{\mathrm{N} / 2-1}\left|c_{1, \mathrm{k}}\right|^{2} \approx \sum_{\mathrm{k}=0}^{\mathrm{N}-1}|F[k]|^{2} \ldots \ldots \ldots \ldots \ldots \ldots \ldots \ldots \ldots \ldots \ldots \ldots \ldots \ldots
\end{aligned}
$$

where $c_{1, \mathrm{k}}$ and $c_{\mathrm{n}, \mathrm{k}}$ are Fourier coefficients when sampling the data at intervals of $T_{\mathrm{s}}$ and $T_{\mathrm{s}} / \mathrm{n}$. Then, a balance relation can be found as

$$
\sum_{\mathrm{k}=0}^{\mathrm{N} / 2-1}\left|c_{1, \mathrm{k}}\right|^{2} \approx \sum_{\mathrm{k}=0}^{\mathrm{nN} / 2-1}\left|c_{\mathrm{n}, \mathrm{k}}\right|^{2}
$$

This equation shows that total power of the signal is saved and the noise scatters into high frequency domain by the oversampling. When the noise power is almost flat in whole bandwidth like a white noise, it holds the following equation;

$$
\forall \mathrm{i} \in[0, \mathrm{nN}-1]\left|c_{\mathrm{n}, \mathrm{i}}\right|^{2} \approx \forall \mathrm{j} \in[0, \mathrm{~N}-1] \frac{1}{\mathrm{n}}\left|c_{1, \mathrm{j}}\right|^{2} .
$$

This also denotes that in-band signal power is reduced $1 / n$ times. This result is an important foothold to handle the transition of the noise.

4.2 Noise Distribution of the System The fast switching become effective to scatter the noise in the wide bandwidth. To check the multirate effect, distributions of quantization errors are confirmed.

In the power system, transistors are driven in a saturation mode to reduce conduction loss. Hence, power should be transferred with a coarse quantizer, which has 2 to at most 17 levels in many cases ${ }^{(14)}$, though pinching a load or cascadeconnection by plural inverters enables to increase the level. For these systems, the pulse-code-modulation (PCM), which is effective for multi level quantizer, is useless for the switching inverter, and a triangular carrier pulse-width-modulation (PWM), which superimposes a dither onto the reference, and a pulse-density-modulation (PDM) are used as alternatives. Now the paper checks noise distributions of an inverters with these modulators. Here, the paper prepares the 2 and 3 level PWM modulators with carrier frequency of $130 \mathrm{kHz}$ and 2 and 3 level PDM modulators whose switching frequency is $100 \mathrm{kHz}$. An input to the inverter is set to $r_{2}(t)$ as the input. 


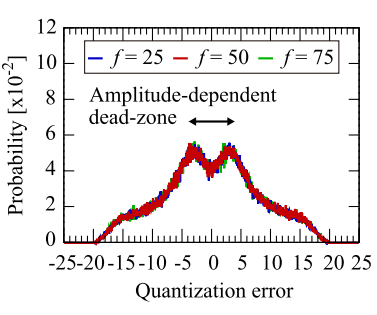

(a) 2-level triangular carrier PWM.
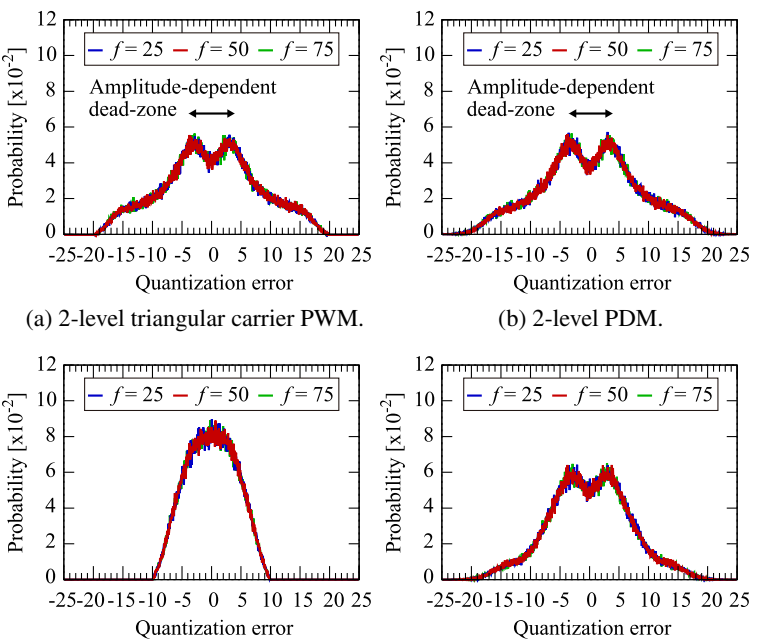

(c) 3-level triangular carrier PWM.

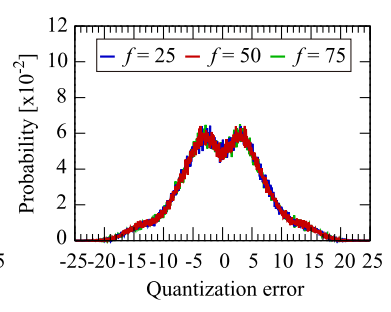

(d) 3-level PDM.

Fig. 1. Histograms of the quantization noise at the input stage at $A=8.0$

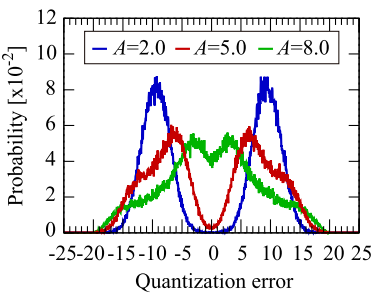

(a) 2-level triangular carrier PWM.

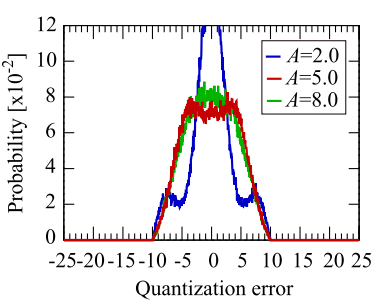

(c) 3-level triangular carrier PWM.

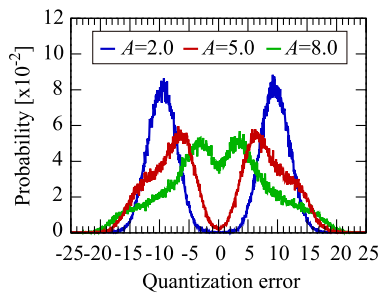

(b) 2-level PDM.

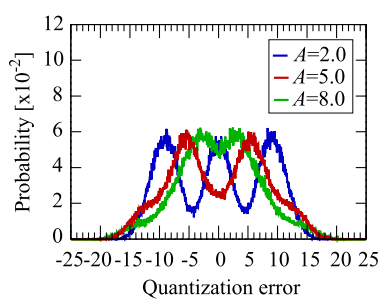

(d) 3-level PDM.

Fig. 2. Histograms of the quantization noise at the input stage at $f=50$

$$
\begin{gathered}
r_{2}(t)=A \sin (2 \pi f t)+p_{2}(t) \cdots \ldots \ldots \ldots \ldots \\
\left(\begin{array}{c}
A=2.0 / 5.0 / 8.0, f=25 / 50 / 75, \text { s.t. } \\
p_{2} \sim \mathcal{N}(0,4)
\end{array}\right)
\end{gathered}
$$

where $\mathcal{N}(a, b)$ stands for the Gaussian distribution with mean of $a$ and variance of $b$. Here, Direct-Current-link (DC-link) voltage is set at $\pm 10 \mathrm{~V}$ and an amplitude of the feedforward reference is $80 \%$ of that voltage. The second term $p_{2}(t)$ is introduced to check tolerance to perturbation.

Probability density functions were derived as shown in Figs. 1 and 2. These figures show the functions for 2 and 3 level PWMs and PDMs, respectively. In the figure (a) and (b), dead-zones due to coarse quantization are confirmed. This shortage is improved by using the 3-level inverter. These results shows that the noise distribution depends on the modulator. Here, it should be noted that the distribution of the quantization error is almost invariant when changing the frequency of the reference wave while fixing the reference amplitude, as shown in Fig. 1. It denotes that the distribution of the quantization noise is invariable regardless of the sampling frequency and the signal is strictly stationary when the
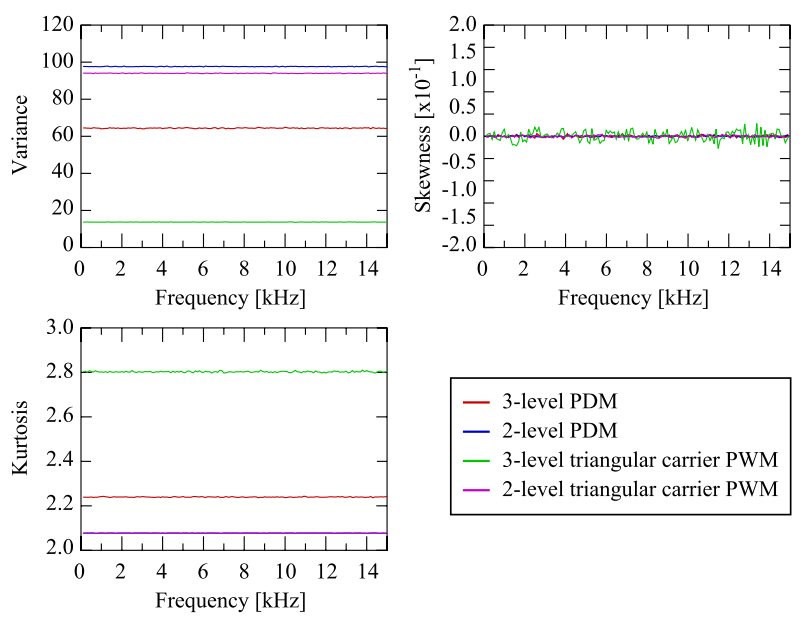

$$
\begin{aligned}
& \text { - 3-level PDM } \\
& \text { - 2-level PDM } \\
& \text { - 3-level triangular carrier PWM } \\
& \text { - 2-level triangular carrier PWM }
\end{aligned}
$$

Fig. 3. Statistical indicators of the quantization noise at the input stage at $A=2.0$
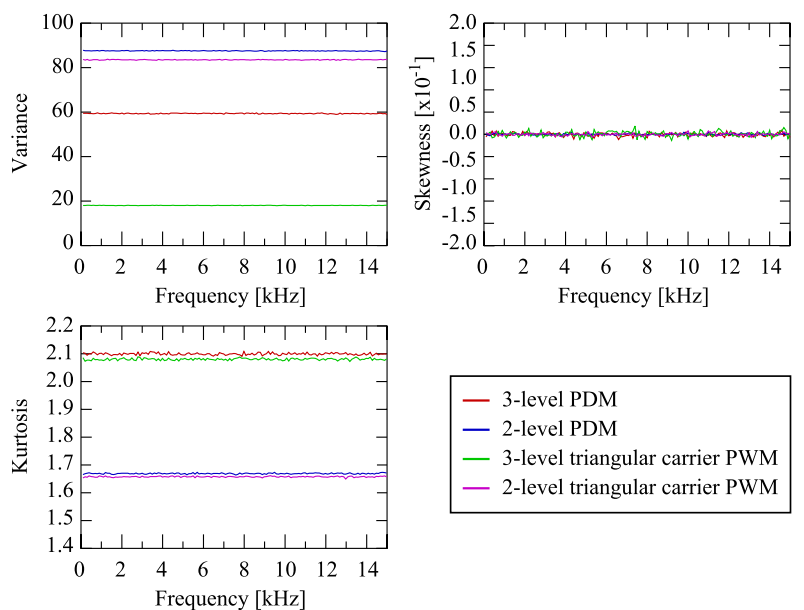

$$
\begin{aligned}
& \text { - 3-level PDM } \\
& \text { - 2-level PDM } \\
& \text { - 3-level triangular carrier PWM } \\
& \text { - 2-level triangular carrier PWM }
\end{aligned}
$$

Fig. 4. Statistical indicators of the quantization noise at the input stage at $A f=5.0$
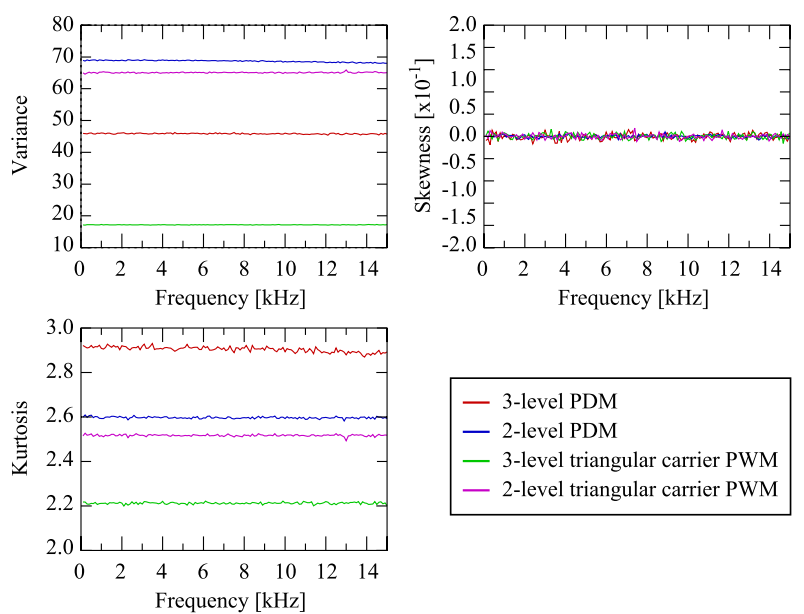

$$
\begin{aligned}
& \text { - 3-level PDM } \\
& \text { - 2-level PDM } \\
& \text { - 3-level triangular carrier PWM } \\
& \text { - 2-level triangular carrier PWM }
\end{aligned}
$$

Fig. 5. Statistical indicators of the quantization noise at the input stage at $A=8.0$

reference amplitude is fixed. This situation is advantaged because the noise power decreases as the switching frequency increases, as shown in (37). However, the quantization noise is not stationary when the reference amplitude changes continuously, as shown in Fig. 2. In these cases, the variance of the quantization error changes along fluctuation of the 
reference amplitude. Then, it is difficult to design a prediction step shown in (29). Here, the paper further examined a stationarity when the reference amplitude does not change. Because of space limitations, histograms for broad frequencies can not be displayed in the paper and the paper checked statistic indicators, variance, skewness, and kurtosis. If those indicators are invariant for whole frequencies, it can be confirmed that stationarity of the system is ensured. Figures 3-5 show graphs of the reference frequency versus the statistic indicators when fixing the reference amplitude to 2.0, 5.0, and 8.0. Red, blue, green, and purple lines indicate values of the 3 level PDM, 2 level PDM, 3 level PWM and 2 level PWM, respectively. In these verifications, mean values of the quantization noise are very close to 0 and the paper regarded and handled that value as 0 . In these figures, flat lines are confirmed, and they show that the quantization noise of all modulator is stationary, regardless the reference amplitudes. These results denotes that the Kalman filter for the power system will show a good effect when the reference amplitude is almost constant.

4.3 Numerical Examination of Noise Effect The paper examined theoretical advantage of the fast input and the distribution of the quantization noise of the power system. Here, the paper checks the multirate effect numerically by using the Monte-Carlo method.

When the system is controlled at the interval of $T_{\mathrm{r} 1}, H_{\mathrm{m}}$ is expressed as $H_{\operatorname{Tr} 1}$. Putting assumption that variance of the process noise $Q=q \in \mathbb{R}$, its variance is expressed as

$$
E\left[v_{\mathrm{hm}}[k] v_{\mathrm{hm}}^{\mathrm{T}}[k]\right]=q H_{\mathrm{Tr} 1} H_{\mathrm{Tr} 1}^{\mathrm{T}}:=Q_{\mathrm{sr}} \cdots \cdots \cdots \cdots
$$

When the sampling rate of the input is $n$ times faster than that of the sensory system, the variance is changed as

$$
\begin{aligned}
E\left[v_{\mathrm{hm}}[k] v_{\mathrm{hm}}^{\mathrm{T}}[k]\right] & =q \sum_{i=0}^{n-1}\left[A_{\mathrm{Tr} 2}^{i} H_{\mathrm{Tr} 2} H_{\mathrm{Tr} 2}^{\mathrm{T}}\left(A_{\mathrm{Tr} 2}^{i}\right)^{\mathrm{T}}\right] \\
& :=Q_{\mathrm{mr}}(n) . \cdots \cdots \cdots \cdots \cdots \cdots
\end{aligned}
$$

Here, derivation of an analytical solution and a comparison are difficult and the paper shows numerical examples. Let us consider a system represented as (1) with parameters

$$
A_{\mathrm{c}}=\left[\begin{array}{cc}
0 & 1 \\
g & -30
\end{array}\right], B_{\mathrm{c}}=\left[\begin{array}{l}
0 \\
1
\end{array}\right]^{\mathrm{T}}, H_{\mathrm{c}}=\left[\begin{array}{l}
0 \\
1
\end{array}\right]^{\mathrm{T}} \ldots \ldots \ldots
$$

Now, $g$ is a system parameter which determines eigenvalues of the state matrix and the $E\left[v_{\mathrm{hm}} v_{\mathrm{hm}}^{\mathrm{T}}\right] \in \mathbb{R}^{2 \times 2}$. The paper compared (40) and (41) focusing on each matrix element while changing the system parameter $g \in[-100:-1]$ and the oversampling ratio $n \in[1: 50]$. Figure 6 shows the comparison of the matrix elements of $Q_{\mathrm{sr}}$ and $Q_{\mathrm{mr}}(n)$. Here, a matrix component $\{i, j\}$ is represented by using an additional term $\{i, j\}$. This figure shows that ratios of the noise variance is proportional to only the oversampling ratio $n$. That is, the a standard deviation of the multirate controller with the oversampling ratio $\sigma_{n}$ approximately meets $\sigma_{n} \propto 1 / \sqrt{n}$.

In order to verify the results, the paper confirmed histograms of the process noises on the system expressed as (1) and (42) with the design parameters $g=-100$ and the noise $v \sim \mathcal{N}(0,100)$. Now, the sampling time of the single-rate controller $T_{\mathrm{r} 1}=10^{-4} \mathrm{~s}$ and the input $u$ is set at 0 to check an effect

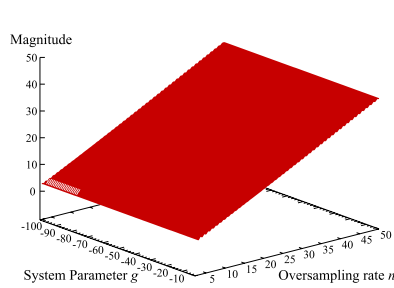

(a) $\mathrm{Q}_{\mathrm{sr}}\{1,1\} / \mathrm{Q}_{\mathrm{mr}}(n)\{1,1\}$

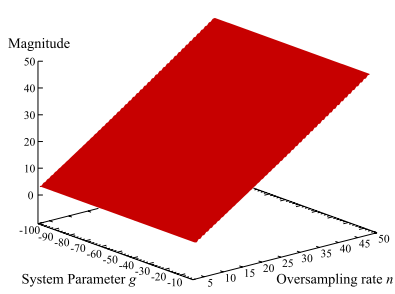

(c) $\mathrm{Q}_{\mathrm{sr}}\{2,1\} / \mathrm{Q}_{\mathrm{mr}}(n)\{2,1\}$

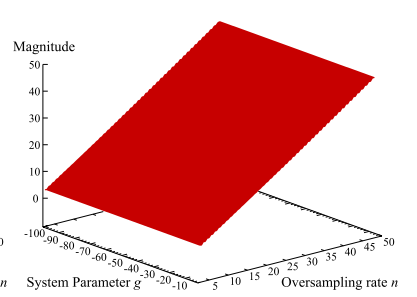

(b) $\mathrm{Q}_{\mathrm{sr}}\{1,2\} / \mathrm{Q}_{\mathrm{mr}}(n)\{1,2\}$

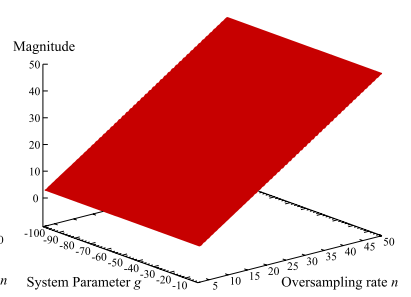

(d) $\mathrm{Q}_{\mathrm{sr}}\{2,2\} / \mathrm{Q}_{\mathrm{mr}}(n)\{2,2\}$
Fig. 6. Ratio of the matrix elements of $Q_{\mathrm{sr}} / Q_{\mathrm{mr}}(n)$

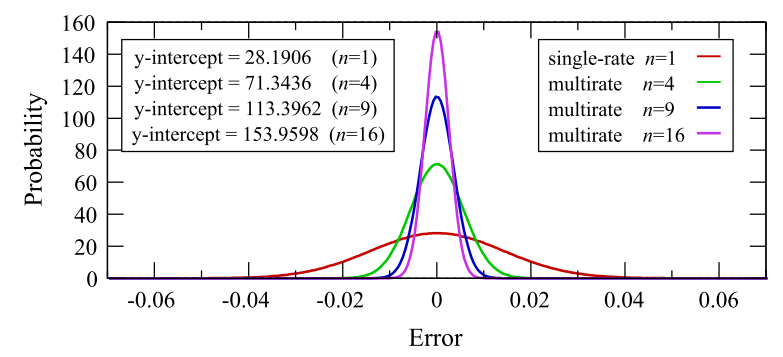

Fig. 7. Histograms of the output sampled at interval $T_{\mathrm{r} 1}$
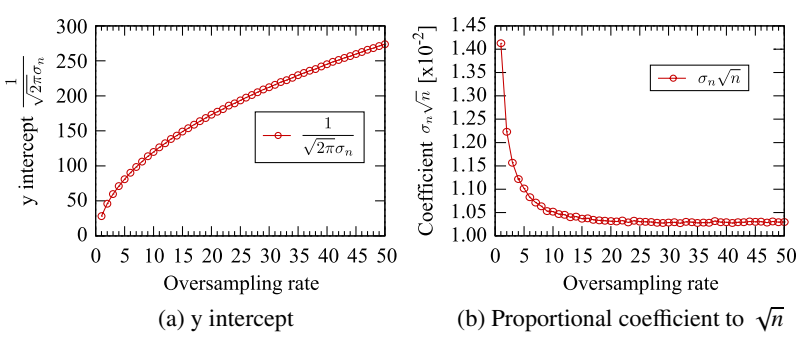

(b) Proportional coefficient to $\sqrt{n}$

Fig. 8. Histograms of the noise effect sampled at intervals of $T_{\mathrm{r} 1}$

of the noise. The effect of the noise is sampled at the interval of $T_{\mathrm{r} 1}$. Figure 7 shows the histograms of the $x\{1,2\}$ which is directly affected by the process noise. The noise variance decreases as $n$ increases. To check a relation between $1 / \sqrt{n}$ and the standard deviation, the paper records them and obtains Fig. 8. This figure shows that the condition $\sigma_{n} \propto 1 / \sqrt{n}$ is established when the oversampling ratio is high.

From these discussions, the equivalent noise variance on the single-rate control system is approximately expressed as

$$
Q_{\mathrm{mr}}(n) \approx Q_{\mathrm{sr}} / n
$$

This result is very similar to the theoretical examine (38). In this derivation, assumptions on the noise distribution is ergodicity and stationarity. When these exist, the proposition (43) can apply to a system with any noise distribution. This equation is rough approximation of (41) and shows an advantage of the high frequency input system. As the variance of the process noise directly affects on update of the Kalman filter as expressed in (29), its value should be small as the estimation error covariance matrix converges into small value. 


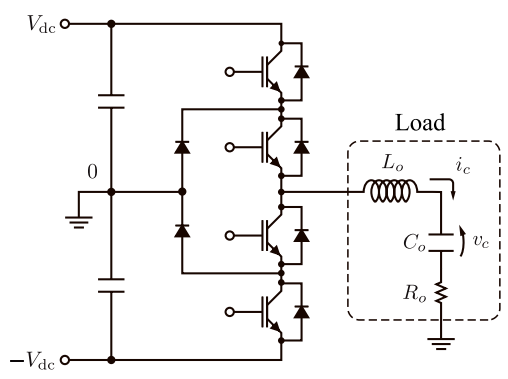

Fig. 9. Diode-clamped 3 level inverter

Table 1. Parameter Descriptions for Numerical Simulation

\begin{tabular}{c|l|c}
\hline \hline Symbol & Description & Value \\
\hline$V_{\mathrm{dc}}$ & DC-link voltage & $50 \mathrm{~V}$ \\
$L_{\mathrm{o}}$ & Load inductance & $100 \mu \mathrm{H}$ \\
$R_{\mathrm{o}}$ & Load resistance & $10 \mathrm{~m} \Omega$ \\
$C_{\mathrm{o}}$ & Load capacitance & $50 \mathrm{mF}$ \\
$i_{\mathrm{c}}$ & Output current & \\
$v_{\mathrm{c}}$ & Output voltage & \\
\hline \hline
\end{tabular}

Namely, increasing the sampling rate of the input system enables to the multirate Kalman filter to estimate a state with low estimation error.

\section{Numerical Simulation}

The paper shows numerical simulation of the multirate Kalman filter for a voltage inverter using a diode-clamped 3 level inverter shown in Fig. 9. Its parameters are described in Table 1. This system is governed by

$$
\begin{aligned}
& \dot{x}=\left[\begin{array}{cc}
0 & 1 / C_{\mathrm{o}} \\
-1 / L_{\mathrm{o}} & -R_{\mathrm{o}} / L_{\mathrm{o}}
\end{array}\right] x+\left[\begin{array}{c}
1 \\
1 / L_{\mathrm{o}}
\end{array}\right] v_{i}\left(\text { s.t. } x \triangleq\left[\begin{array}{c}
v_{\mathrm{c}} \\
i_{\mathrm{c}}
\end{array}\right]\right) \\
& \text {................... (44) } \\
& y=\left[\begin{array}{ll}
1 & 0
\end{array}\right] x+w(w \sim \mathcal{N}(0,1)) .
\end{aligned}
$$

A process noise is defined as an error between desired input voltage and practical one, namely $v(t)=v_{\mathrm{i}}(t)-v_{\mathrm{c}}^{\mathrm{ref}}(t)$. The paper assumed an observation noise closely follows a normal distribution. The input voltage switches with frequency of $50 \mathrm{kHz}$ and the output voltage is obtained by using a $5 \mathrm{ksps}$ 12 bit A/D converter. Desired voltage output was set as

$$
v_{\mathrm{c}}^{\mathrm{ref}}(t)=40 \sin (120 \pi t),
$$

whose amplitude is $80 \%$ of the DC-link voltage. The reference voltage was modulated by the triangular carrier PWM with a carrier frequency of $20 \mathrm{kHz}$. Then, the variance of the process noise was obtained as $\operatorname{Var}[v[k]]=4.7071 \times 10^{2}:=\sigma_{\mathrm{v}}^{2}$. This value was calculated based on discussion in the section 3. As a result, an equivalent noise variance is expressed as

$$
\operatorname{Var}\left[v_{\mathrm{hm}}\right]=\left[\begin{array}{cc}
0.024474 & 1.821245 \\
1.821245 & 182.138849
\end{array}\right]
$$

The multirate Kalman filter is able to be designed with above variance and non-stochastic input term observed at interval of $200 \mu$ s, as formulated in (13). Now a number of design-DOFs in tracking is 5 since an oversampling ratio and an order of the system are 10 and 2. The redundant DOFs are used for the input softening in this time.

The paper conducted two verifications. Case I is validation
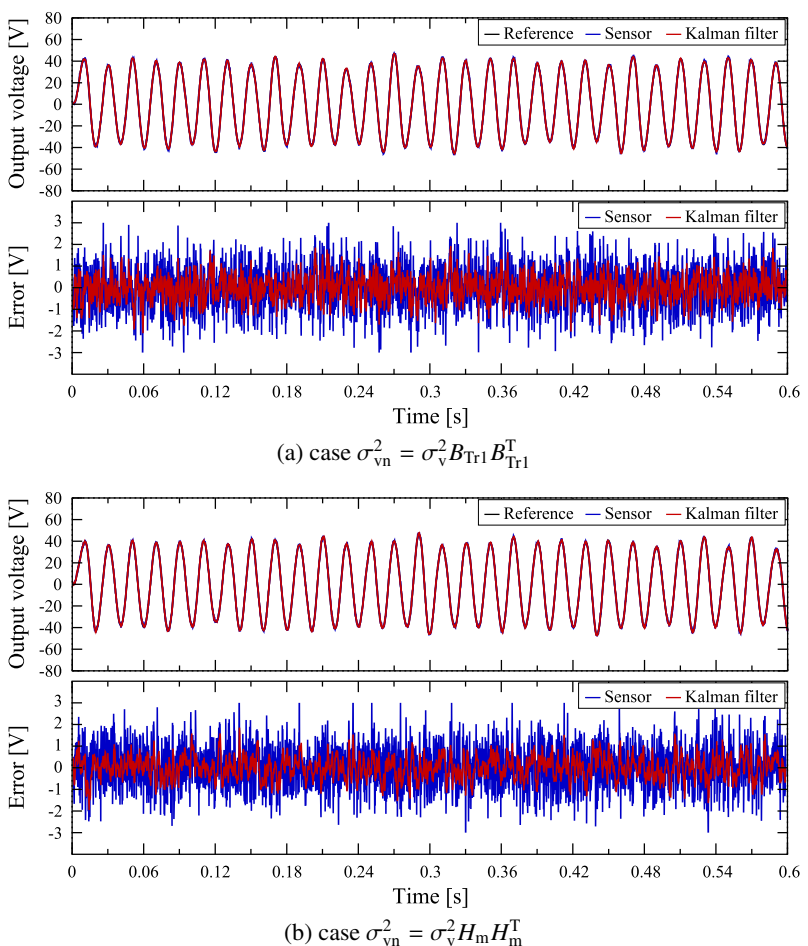

Fig. 10. Output waveforms and sensing/estimation errors in the case I

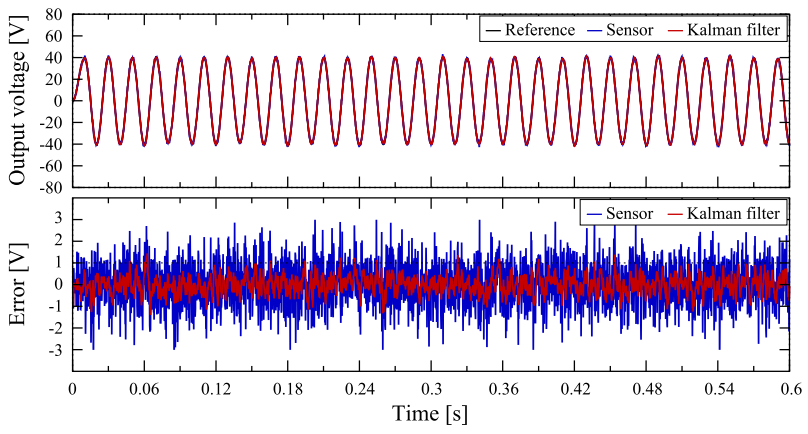

Fig. 11. Output waveforms and sensing/estimation errors in the case II

of the multi-rate Kalman filter when the process noise $v$ follows a normal distribution $\mathcal{N}\left(0, \sigma_{\mathrm{v}}^{2}\right)$. The input was designed as $v_{\mathrm{i}}=v_{\mathrm{c}}^{\mathrm{ref}}+v$ and the paper compared cases that the variances of the process noise $\sigma_{\mathrm{vn}}^{2}$ were set at $\sigma_{\mathrm{v}}^{2} H_{\mathrm{m}} H_{\mathrm{m}}^{\mathrm{T}}$ and $\sigma_{\mathrm{v}}^{2} B_{\mathrm{d} 1} B_{\mathrm{d} 1}^{\mathrm{T}}$, which considered noise shaping of the multirate controller or not. Case II is application for the switching inverter. Conditions for this simulation is the same as discussed in above. In whole simulation, a true value and the estimated values are compared and estimation errors are evaluated.

Figure 10 shows results in the case I. In the case setting $\sigma_{\mathrm{vn}}^{2}=\sigma_{\mathrm{v}}^{2} B_{\mathrm{d} 1} B_{\mathrm{d} 1}^{\mathrm{T}}$, a variance of the estimation error is 0.5870 , which denotes $41.30 \%$ decrease of the variance of the observation noise. On the other hand, the case setting $\sigma_{\mathrm{vn}}^{2}=\sigma_{\mathrm{v}}^{2} H_{\mathrm{m}} H_{\mathrm{m}}^{\mathrm{T}}$ shows a variance of the estimation error of 0.4891 , which denotes $51.19 \%$ decrease of the variance of the observation noise. This results show an effectiveness of the rigorous multirate-design for the state estimation.

Figure 11 shows the results in the case II. In this simulation, a variance of the estimation error is 0.4220 , which denotes $67.80 \%$ decrease of the variance of the observation noise. 


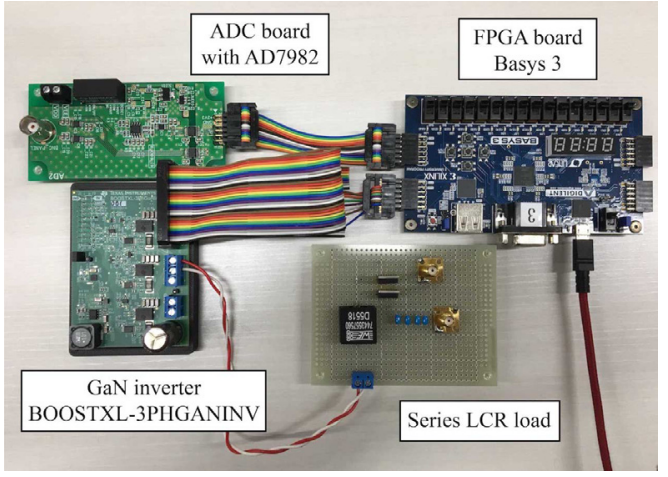

Fig. 12. Experimental setup

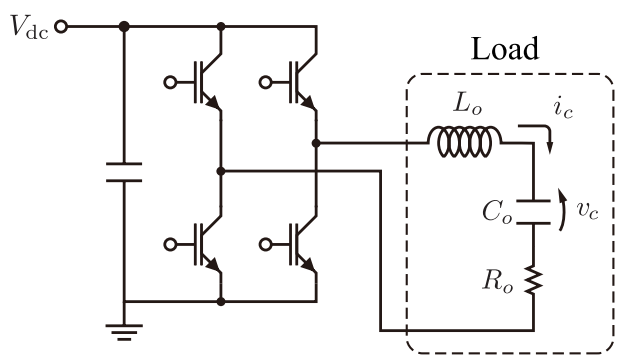

Fig. 13. Equivalent circuit of the experimental setup

This result shows that the multi-rate Kalman filter has possibility to provide good estimation for the voltage inverter. As denoted in (43), more suppression of the estimation error requires increasing of the switching frequency or expanding the level of the inverter as discussed in the section 3 . Note that excessive increase of switching frequency should be avoided since it causes extra noises due to voltage clamp in a deadtime or reverse-recovery of diodes ${ }^{(15)(16)}$.

\section{Experiments}

The paper conducted the experiments of estimating a state by using the multirate Kalman filter. In the experiments, a sinusoidal wave of was input to a pulse modulator and the inverter output voltage to a series LCR load. Because the paper focuses on the theoretical verification, such simple load was prepared. Then, the voltage across the capacitor is measured through the A/D converter. Since true values of the state can not be obtained in the experiments, the paper regarded the data from an $\mathrm{A} / \mathrm{D}$ converter as the true value and then that value plus noise as the sensor output.

6.1 Experimental Setup An experimental setup is shown in Fig. 12. A controller was designed on an FPGA board (Basys 3; Digilent) which has a Xilinx XC7A35T and clock source of $100 \mathrm{MHz}$. A gallium-nitride highelectron-mobility-transistor (GaN-HEMT) based 3-phase inverter board (BOOSTXL-3PHGANINV; Texas Instruments) was prepared and 2-phases were used to construct an $\mathrm{H}$ bridge. Since the GaN-HEMTs can perform fast switching, non-ideality such as distortion at switching is reduced and the dead-time is shortened. Then, the dead-time for each legs was set to $100 \mathrm{~ns}$. Furthermore, the reverse-conducting characteristics mitigates a voltage clamp in the dead time without free-wheel diodes. In this time, DC-link voltage was set to $8.0 \mathrm{~V}$. A reference amplitude and a reference frequency were set to $2.0 \mathrm{~V}$ and $500 \mathrm{~Hz}$. The reference wave was input

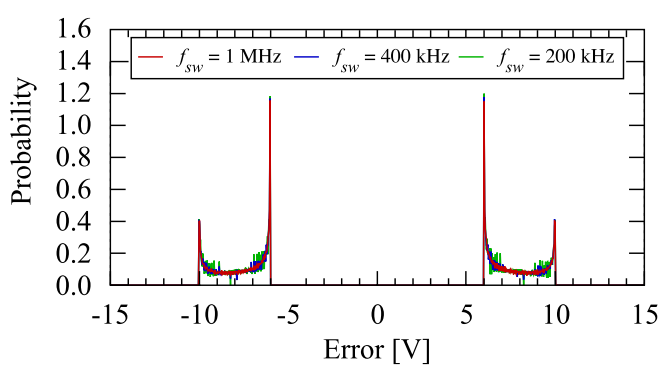

Fig. 14. Histograms of the quantization error of each switching frequency

Table 2. Parameter Descriptions for Experiments

\begin{tabular}{c|l|c}
\hline \hline Symbol & Description & Value \\
\hline$V_{\mathrm{dc}}$ & DC-link voltage & $8.0 \mathrm{~V}$ \\
$L_{\mathrm{o}}$ & Total inductance of the circuit & $6.231 \mu \mathrm{H}$ \\
$R_{\mathrm{o}}$ & Total resistance of the circuit & $1.542 \Omega$ \\
$C_{\mathrm{o}}$ & Total capacitance of the circuit & $17.498 \mu \mathrm{F}$ \\
$v_{\mathrm{c}}$ & Output voltage & \\
\hline \hline
\end{tabular}

to the 2nd-order delta-sigma modulator and converted into gate signals for the GaN-HEMTs. Switching frequencies of the delta-sigma modulator were set to $200 \mathrm{kHz}, 400 \mathrm{kHz}$ and 1.0 MHz. Here, an ideal distribution of the quantization error is shown in Fig. 14. Histograms draw similar form while distortion can be seen as switching frequency decreases. All variances for each switching frequency converged to 62.000. A load installed on the output stage of the inverter is composed of a choke coil, fixed thick-film resistors and multilayer ceramic capacitors, to establish linearity within wide bandwidth. For the theoretical verification, non-ideality on the circuit was removed as possible. Circuit parameters were measured by an LCR meter (IM3536; HIOKI) and listed in Table 2. A state-space equation of this system is the same as (44) and (45). The voltage across the capacitor is measured through the A/D conversion board which has 18 bit A/D converter (AD7982; Analog Devices). The paper regarded the data from an A/D converter $y$ as the true value and then that value $y$ plus noise $w_{\mathrm{p}}$, namely $y+w_{\mathrm{p}}$, as the sensor output. The noise $w_{\mathrm{p}}$ was generated by using the Box-Muller's method and its variance was set to 0.5 .

6.2 Experimental Results Sensor outputs, sensor output with noise versus filtered state, and noise versus estimation errors for each switching frequency are shown in Figs. 15-17. As the switching frequency increases, distortion on the output decreases. The power spectrum of the voltage across the capacitor is shown in Fig. 18. Noise floors of the systems with switching frequency of $400 \mathrm{kHz}$ and $1.0 \mathrm{MHz}$ are almost flat, while the other has a soaring floor. This difference is caused by the distortion confirmed in Fig. 14. Compared with the system with the switching frequency of $400 \mathrm{kHz}$ and $1.0 \mathrm{MHz}$, noise suppression of $8.0 \mathrm{~dB} \approx 20 \log _{10} 2.5$ can be confirmed in whole frequency. This result matches to (38). Then, the relation between the oversampling and the noise reduction is partially confirmed in the experiments. Checking the filtered states, reductions of variance of the estimation error are $25.9 \%$ for $200 \mathrm{kHz}$, $41.7 \%$ for $400 \mathrm{kHz}, 59.1 \%$ for $1.0 \mathrm{MHz}$ switching system, respectively. Thus, the variance of the estimation error increases as the switching frequency decreases. This is because 


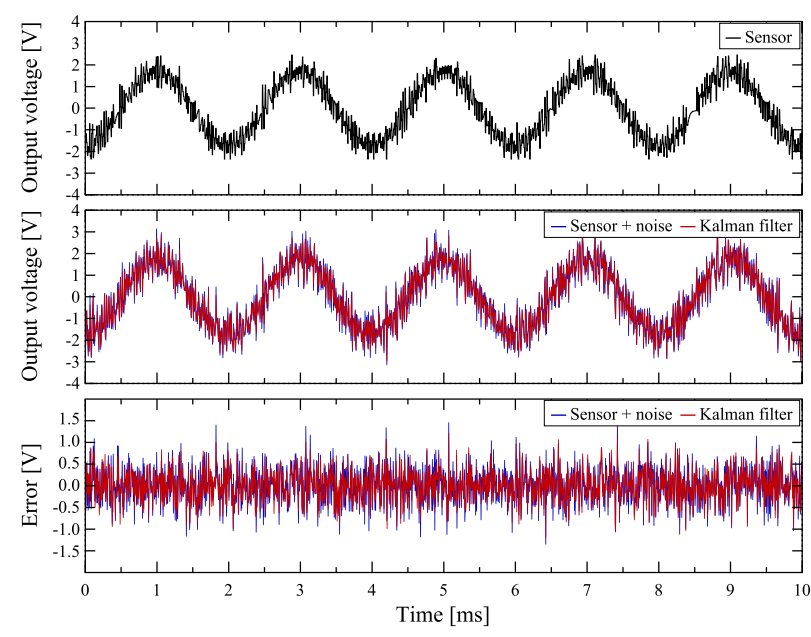

Fig. 15. Output waveforms and sensing/estimation errors when the switching frequency is $200 \mathrm{kHz}$

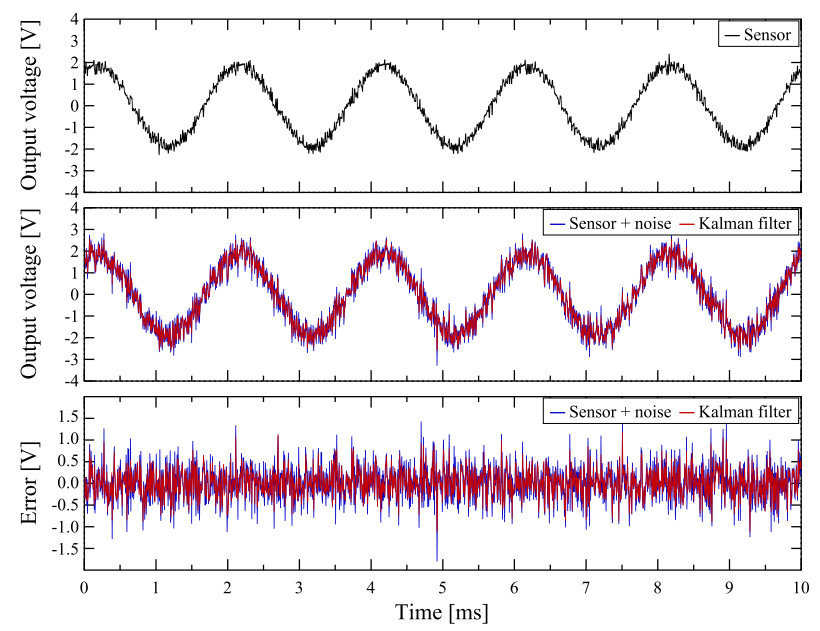

Fig. 16. Output waveforms and sensing/estimation errors when the switching frequency is $400 \mathrm{kHz}$

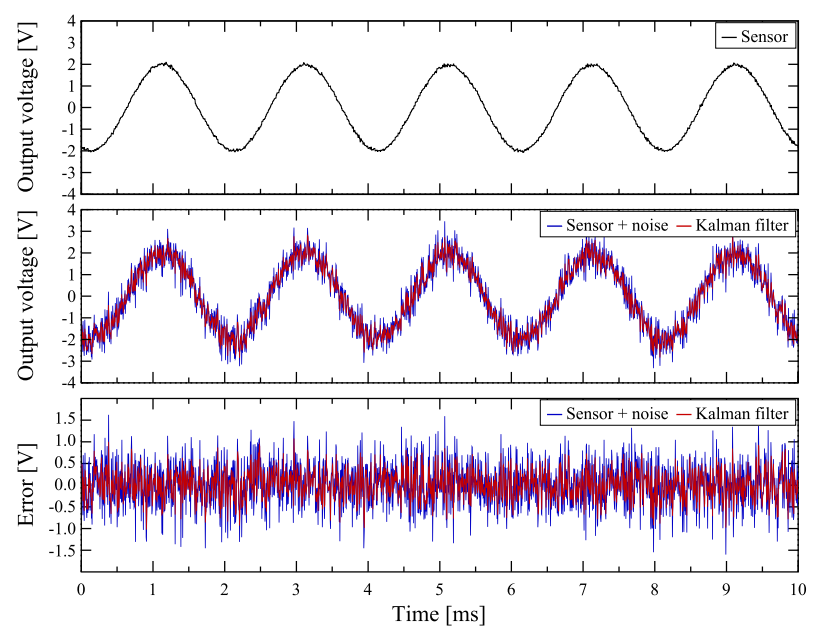

Fig. 17. Output waveforms and sensing/estimation errors when the switching frequency is $1.0 \mathrm{MHz}$

the Kalman filter is low-pass filter whose cut-off frequency is determined by the reliability of the input/output system, as shown in (27). With the low switching-frequency, variance of the process noise is large and the Kalman filter tends to trust a sensor value and then the sensor noise is mistaken as

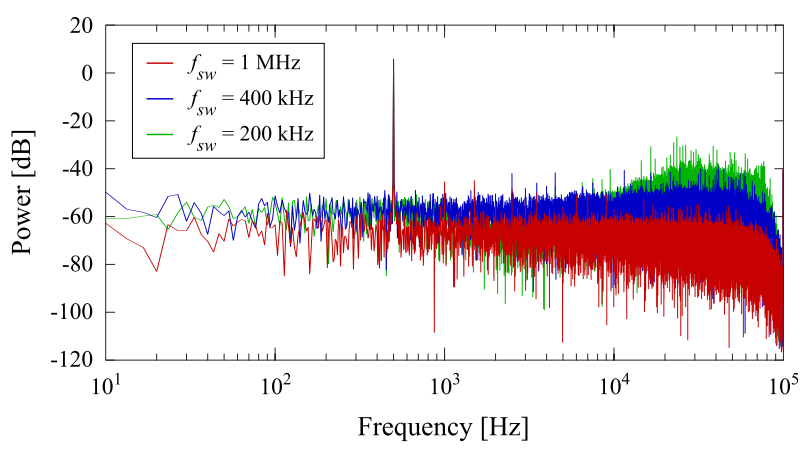

Fig. 18. Power spectra of the sensor data

the true value. It is desirable to set the switching frequency to high from viewpoints of reduction of distortion on the output and variance of the process noise. For such situation, design methodology of the multirate Kalman filter should come in useful.

\section{Conclusion}

This paper shows the multirate Kalman filter design for the system with the fast input system. In design, the multirate controller is modeled as is the case for the singlerate controller by lifting method and the equivalent noise on the single-rate one is defined. The problem of the multirate Kalman filter comes down into how to define the process noise. Then, the paper examined the multirate effects against the noise and evaluated them by using the Monte Carlo method. From whole discussion, the multirate Kalman filter is designed for the voltage inverter and the numerical simulation and experiments shows the validity of the designed filter.

\section{Acknowledgment}

This work was partially supported by JSPS KAKENHI Grant Number $18 \mathrm{H} 03784$.

\section{References}

( 1 ) H. Okajima, M. Honda, R. Yoshino, and N. Matsunaga: "A Design Method of Delta-Sigma Data Conversion System with Pre-Filter", SICE Journal of Control, Measurement, and System Integration, Vol.8, No.2, pp.154-160 (2015)

( 2 ) M. Suzuki and M. Hirata: "Exact Linearization of Third-order Systems with Pulse-width-modulation-type Inputs", Nonlinear Theory and Its Applications, IEICE, Vol.9, No.4, pp.204-217 (2018)

( 3 ) R. Mitova, R. Ghosh, U. Mhaskar, D. Klikic, M.X. Wang, and A. Dentella: "Investigations of 600-V GaN HEMT and GaN Diode for Power Converter Applications", IEEE Transactions on Power Electronics, Vol.29, No.5, pp.2441-2452 (2014)

( 4 ) E. Gurpinar and A. Castellazzi: "Single-Phase T-Type Inverter Performance Benchmark Using Si IGBTs, SiC MOSFETs, and GaN HEMTs", IEEE Transactions on Power Electronics, Vol.31, No.10, pp.7148-7160 (2016)

( 5 ) K. Sakata and H. Fujimoto: "Perfect Tracking Control of Servo Motor Based on Precise Model with PWM Hold and Current Loop", Power Conversion Conference, pp.1612-1617 (2007)

( 6 ) H. Ohkubo, T. Shimono, and N. Motoi: "The Performance Validation of Disturbance Observer Based on Comparison between Motion Control Frequency and Current Control Frequency", The 12th IEEE International Workshop on Advanced Motion Control, pp.1-6 (2012)

( 7 ) Y. Yokokura and K. Ohishi: "FPGA-Based Broadband Current Control of a Linear Motor with Class-G Power Amplifiers", IEEE International Conference on Mechatronics, pp.528-533 (2013)

( 8 ) A. Smyth and M. Wu: "Multi-rate Kalman Filtering for the Data Fusion of Displacement and Acceleration Response Measurements in Dynamic System Monitoring", Mechanical Systems and Signal Processing, Vol.21, No.2, pp.706-723 (2007) 
( 9 ) B.M. Nguyen, Y. Wang, H. Fujimoto, and Y. Hori: "Advanced Multi-rate Kalman Filter for Double Layer State Estimator of Electric Vehicle Based on Single Antenna GPS and Dynamic Sensors", The 6th IFAC Symposium on Mechatronic Systems, Vol.46, No.25, pp.437-444 (2013)

(10) A. Liu, W.-A. Zhang, M.Z.Q. Chen, and L. Yu: "Moving Horizon Estimation for Mobile Robots With Multirate Sampling", IEEE Transactions on Industrial Electronics, Vol.64, No.2, pp.1457-1467 (2016)

(11) P. Albertos: "Block multirate input-output model for sampled-data control systems", IEEE Transactions on Automatic Control, Vol.35, No.9, pp.10851088 (1990)

(12) H. Fujimoto and A. Kawamura: "Perfect Tracking Digital Motion Control Based on Two-Degree-of-Freedom Multirate Feedforward Control", The 5th IEEE International Workshop on Advanced Motion Control, pp.322-327 (1998)

(13) M. Tomizuka, D.M. Auslander, and Y. Takahashi: "Simple Finite-Time Settling Control and Manipulated-Variable Softening for Reverse Reaction, Overshoot, and Oscillatory Processes", Transactions on ASME, Journal of Dynamic Systems, Measurement and Control, No.100, Vol.1, pp.50-58 (1978)

(14) M. Sarbanzadeh, E. Babaei, M.A. Hosseinzadeh, and C. Cecati: "A New Sub-Multilevel Inverter with Reduced Number of Components", 42nd Annual Conference of the IEEE Industrial Electronics Society, pp.3166-3171 (2016)

(15) N.K. Trung, T. Ogata, S. Tanaka, and K. Akatsu: "Analysis and PCB Design of Class D Inverter for Wireless Power Transfer Systems Operating at 13.56 MHz", IEEJ Journal of Industry Applications, Vol.4, No.6, pp.703-713 (2015)

(16) H. Kurumatani and S. Katsura: "GaN-HEMT-Based Three Level T-type NPC Inverter Using Reverse-Conducting Mode in Rectifying", 2017 IEEE 26th International Symposium on Industrial Electronics (ISIE), pp.19411946 (2017)
Hiroki Kurumatani (Member) received the B.E. degree in system de-

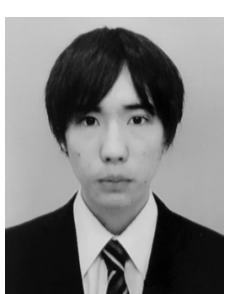
sign engineering and the M.E. degree in integrated design engineering from Keio University, Yokohama, Japan, in 2015 and 2017, respectively. Since 2015, he has been a Ph.D. course student at Keio University, Yokohama, Japan. His research interests include system design of electromechanical integrated systems and industrial electronics. He is a Member of IEEJ, as well as IEEE.

Seiichiro Katsura (Senior Member) received the B.E. degree in sys-

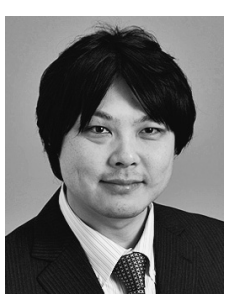
tem design engineering and the M.E. and Ph.D. degrees in integrated design engineering from Keio University, Yokohama, Japan, in 2001, 2002 and 2004, respectively. From 2003 to 2005, he was a Research Fellow of the Japan Society for the Promotion of Science (JSPS). From 2005 to 2008, he worked at Nagaoka University of Technology, Nagaoka, Niigata, Japan. Since 2008, he has been at Keio University, Yokohama, Japan. In 2017, he was a Visiting Researcher with the Laboratory for Machine Tools and Production Engineering (WZL) of RWTH Aachen University, Aachen, Germany. His research interests include applied abstraction, human support, data robotics, wave system, systems energy conversion, and electromechanical integration systems. Prof. Katsura serves as an Associate Editor of the IEEE Transactions on Industrial Electronics. He was the recipient of the IEEJ Distinguished Paper Awards in 2003 and 2017, IEEE Industrial Electronics Society Best Conference Paper Award in 2012, and JSPS Prize in 2016. 